\title{
THE INTERACTION OF BINARY SYSTEMS WITH ACCRETION DISKS
}

\author{
Manuel Ortega-Rodríguez ${ }^{1}$
}

\section{RESUMEN}

Se estudia las fluctuaciones (temporales) en la radiación saliente de discos de acreción en sistemas binarios, con el objeto de determinar las propiedades de dichos sistemas (comparando con las observaciones), tales como el momento angular de la estrella compacta alojada en el disco. Después de una discusión de los efectos de la estrella compañera se presenta una predicción de la frecuencia de la radiación saliente (modulada) de rayos $\mathrm{X}$.

\section{ABSTRACT}

We study the (time) fluctuations in the outgoing radiation of accretion disks in binary systems in order to obtain properties of those systems (via comparison with observations), such as the angular momentum of the compact star within the disk. The effects of the companion star are discussed, and a prediction is made on the frequency of the outgoing (modulated) X-ray radiation.

\section{Key Words: ACCRETION, ACCRETION DISKS - BLACK HOLE PHYSICS - GRAVITATION - HYDRODYNAMICS - RELATIVITY}

\section{INTRODUCTION}

Accretion disks can be used as probes to study the properties of stars and binary systems. Since they can become observable under certain conditions (e.g., the strong gravitational fields of compact objects), the properties of an X-ray binary system, for example, can be obtained via the study of fluctuations in time of the disk's outgoing radiation. This field is known as diskoseismology (Wagoner 1999).

The time-modulated X-rays of Galactic binary systems, e.g. the microquasar GRS $1915+105$, have been observed by the Rossi X-ray Timing Explorer. Comparison of theoretical models with observational data yields several properties of the system. For example, the angular momentum of a black hole in an X-ray binary can be found in principle by this method.

Since diskoseismology is not limited to X-ray binaries, or to binary systems, another way in which accretion disks may be used to probe binary systems is by comparing the diskoseismic results in these systems with those in disks around single stars.

\section{OVERVIEW}

The present work concerns theoretical modeling of accretion disks. We deal mainly with X-ray binaries, in which a main sequence star provides gas for accretion onto a black hole. We study only the effects of the binary on the disk, and deal with a particular property of the disk: the frequency of its

\footnotetext{
${ }^{1}$ Escuela de Física, Universidad de Costa Rica, San José, Costa Rica. email: manuel@gravity.stanford.edu
}

small normal-mode oscillations. We have neglected the effects of the companion star (see, however, §5).

\section{ACCRETION DISKS}

Under certain conditions a binary system can host an accretion disk. If the Roche lobe of one of the stars is filled, material can flow to the other star. A disk will be formed if the gas has angular momentum. Turbulent viscosity generates heat and is responsible for the (radial) outward transport of angular momentum. The radiation comes out as Xrays for accretion onto Galactic black holes (and as visible radiation for supermassive black holes).

Both observations and theory suggest that there are several types of state for the equilibrium accretion disk, the main ones being: a) a geometrically thin, radiatively efficient disk, the so-called 'standard' $\alpha$ accretion disk (Shakura \& Sunyaev 1973; Novikov \& Thorne 1973); b) a geometrically thick, radiatively inefficient disk, dubbed ADAFs for 'advectively dominated accretion flows' (e.g. Abramowicz et al. 1988; Abramowicz et al. 1995). The actual state is unknown. One possibility is a limit cycle in which the disk goes through both types periodically.

\section{NORMAL MODES}

Diskoseismology, the study of linearized perturbations in accretion disks, obtains a normal-mode eigenfrequency spectrum (in which all particles oscillate with the same frequency) when boundary conditions are imposed. By relating these eigenfrequencies to the properties of the compact object within the 
disk (its mass or angular momentum), these properties can be in principle determined.

This field was developed in Nowak \& Wagoner (1991; 1992), inspired by the suggestion of Kato \& Fukue (1980) that normal modes can be trapped in accretion disks around compact objects. Perez et al. (1997), Silbergleit, Wagoner \& Ortega-Rodríguez (2001), and Ortega-Rodríguez, Silbergleit \& Wagoner (2002) discuss the relativistic case.

The starting point is the hydrodynamical equation of the gas in the disk:

$$
\nabla_{a} T^{a b}=0,
$$

where $\nabla_{a}(a=0,1,2,3)$ is the covariant derivative, and where

$$
T^{a b}=\rho u^{a} u^{b}+p q^{a b}
$$

is the stress-energy tensor of a perfect relativistic fluid ( $\rho$ and $u^{a}$ stand for density and 4-velocity, while $q^{a b} \equiv g^{a b}+u^{a} u^{b}$, and $g^{a b}$ refers to the Kerr metric).

After perturbing equation (1) adiabatically, the problem can be split by separation of variables into two coupled differential equations, one radial and one vertical, which can be solved using the WKB method to yield the eigenfrequencies, which depend on the mass and angular momentum of the compact star.

This procedure reveals the existence of three types of mode. G-modes occupy the hottest (and most visible) part of the disk, and are mainly radial oscillations in which pressure acts against gravitational restoring forces; $p$-modes are mainly radial oscillations in the inner- and outermost parts of the disk, as well as in the disk as a whole, in which pressure acts with gravity; c-modes are vertical incompressible oscillations in the innermost part of the disk, in which pressure acts against gravity.

\section{ON THE POSSIBLE EFFECTS OF THE COMPANION}

Accretion disks exist both around single (protostars, AGNs) and binary (X-ray Galactic binary, supermassive binary) systems. In the case of X-ray binaries, for example, the existence of the disk is due precisely to the gas shed by the companion.

We now consider whether the companion plays a further rôle in the system (other than providing mass). When there was a problem finding a mechanism for angular momentum transfer in accretion disks (molecular viscosity not being a big enough effect), a possible explanation was sought in the companion's tidal effects on the disk. This was inadequate, however (Terquem 2002), and it is now believed that turbulent viscosity due to a MHD insta- bility is the responsible mechanism (Balbus \& Hawley 1991).

The main effect of the companion on the disk's outgoing radiation may be the hot spot produced when gas from the companion impacts the disk. This spot would enhance the outer p-modes, which are normally not visible for single-star accreting systems.

\section{DISCUSSION}

Relativistic diskoseismology, the theoretical study of small normal-mode oscillations in accretion disks around compact stars, provides a potentially powerful method to measure, e.g., the angular momentum of a black hole. In addition, it can probe general relativity in a strong field regime.

The method can be applied to any disk provided the object within is compact. Thus, in addition to Xray binaries, it could be applied to AGNs. Diskoseismology may be used to study binary systems since its predictions depend on the existence of a hot spot.

Based on diskoseismology, we predict that ( $p$ mode) quasi-periodic oscillations with frequencies $\sim 0.1 \mathrm{~Hz}$ might be observable in accretion disks in binary systems, due to a companion-induced hot spot. (See Fig. 15 in Ortega-Rodríguez et al. 2002).

The present work was supported by grant 075 2002 (Incentivos) of Ministerio de Ciencia y Tecnología, Costa Rica and by grant 112-A3-078 of Vicerrectoría de Investigación, Universidad de Costa Rica, to the author.

\section{REFERENCES}

Abramowicz, M. A., Czerny, B., Lasota, J.-P., \& Szuszkiewicz, E. 1988, ApJ, 332, 646

Abramowicz, M. A., Chen, X., Kato, S., Lasota, J.-P. \& Regev, O. 1995, ApJ, 438, L37

Balbus, S. A., \& Hawley, J. F. 1991, ApJ, 376, 214

Kato, S., \& Fukue, J. 1980, PSAJ, 32, 377

Novikov, I. D. \& Thorne, K. S. 1973, in Black Holes, ed. C. DeWitt \& B. S. DeWitt (New York: Gordon and Breach), 343

Nowak, M. A., \& Wagoner, R. V. 1991, ApJ, 378, 656

Nowak, M. A., \& Wagoner, R. V. 1992, ApJ, 393, 697

Ortega-Rodríguez, M., Silbergleit, A. S., \& Wagoner, R. V. 2002, ApJ, 567, 1043

Perez, C. A., Silbergleit, A. S., Wagoner, R. V., \& Lehr, D. E. 1997, ApJ, 476,589

Shakura, N. I. \& Sunyaev, R. A. 1973, A\&A, 21, 127

Silbergleit, A. S., Wagoner, R. V., \& Ortega-Rodríguez, M. 2001, ApJ, 548, 335

Terquem, C. 2002, in EAS Pub. Ser. 3, Star Formation and the Physics of Young Stars, ed. J. Bouvier \& J.-P. Zahn, 203

Wagoner, R. V. 1999, Phys. Rep., 311, 259 